\title{
Combatting
} internet time shifters

Michael Schapira

The Hebrew University of Jerusalem

Combatting internet time shifters

The Network Time Protocol (NTP) synchronises computer systems across the internet (typically within a few
milliseconds of Coordinated Universal Time) and is ubiquitously deployed. Many internet applications, including TLS certificates, internet naming addressing (via DNS and DNSSEC internet routing security mechanisms
(namely, RPKI), Kerberos, financial (namely, RPKI), Kerberos, financia
services, and many others, crucially rely services, and many others, crucially rely
on NTP for both correctness and safety.

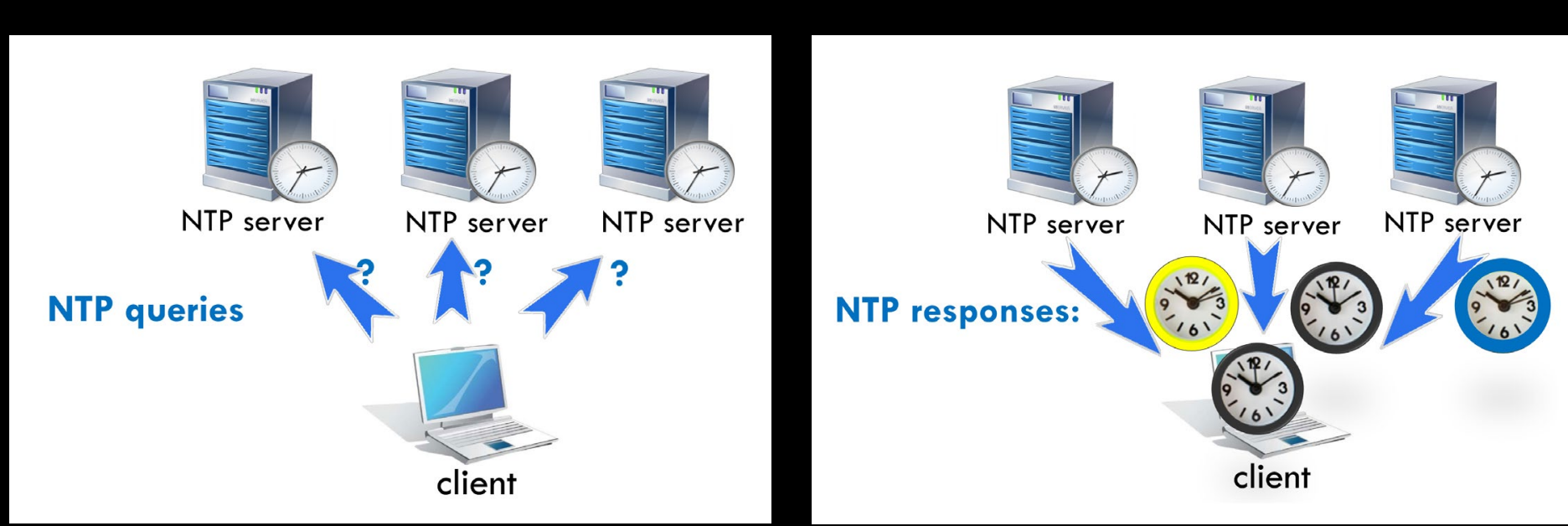

Figure 1a and 1b: An NTP chient (a) exchanges messages with time servers and then (b) receives current clock readings at the time servers and estimates network delay for each time server
How does NTP work? 'offset' for each time server, i.e. the client's local clock and the time server's local clock. To update its local time, the client feeds the resulting offsets into an algorithm that discards outliers and computes, from the 'surviving' offsets, a new time to update the local clock to.

\section{NTP servers and the} NTP server pool

The NTP Pool Project centralises access to a pool of thousands of volunteered NTP clock readings, the client computes the servers across different countries an organisational domains. The NTP serv pool assigns time servers to NTP clients based on client geolocation and balances load across its servers. By default, the NTP Pool Project is used by most, if not all, open-source OS (operating systen) distributions-including all major Lin distributions-router vendors, home automation systems, security cameras, household appliances, and more.

\section{NTP is vulnerable to} time-shifting attacks

Similarly to other internet infrastructure components (for example, TCP/IP, BGP, DNS), NTP was designed withou security in mind. NTP's design thu reflects the need to achieve correctness in the presence of inaccurate clocks ('falsetickers'), assumed to be fair// rare, as opposed to designated attack by powerful and strategic adversaries. Consequently, NTP is alarming/V vulnerable to attacks.

We consider time-shifting attacks, in which an attacker shifts the local time at the NTP client forward/backwards. Recall that the local time at a client is determined based on the clock readings received from the time servers the client interacts with and the delay with respect to these time servers, as estimated by the client. By reporting false clock readings at client. By reporting false clock readings at delay between the client and the time servers, an attacker can induce wron decisions at the NTP client. In particular, if the attacker has sufficient presence in the set of time servers with which an NTP $+$ $+$ $+$



client communicates, it can stealthily shift time at the client by repeatedly pushing the client further away from client. Such attacks can be launched, for example, by a man-in-the-middle (MitM) attacker capable of intercepting and tampering with NTP messages between the client and (a significant subset of the) time servers, or by an attacker in direct control of (a subset of) the NTP servers themselves. See Figure 2

Recently introduced patches to NTP's implementation eliminate/mitigate some off-path attacks and implementation flaws. Yet, MitM attackers-let alone attackers in direct control of time servers-are often deemed too strong

"MitM attackers-let alone

attackers in direct control of time servers-are often deemed too strong to protect against." protect against. The cure to some of 作 NTP traffic between clients and servers. owever, even ubiquitous encryption and authentication is insufficient for fully protecting NTP time synchronisation from a MitM attacker capable merely of delaying and replaying packets and, of course, from malicious time servers.

ust how vulnerable is NTP to malicious timeservers?

Our focus is on investigating and addressing NTP's vulnerability to trategic attacks by malicious time servers. The natural starting point for his investigation is the NTP Pool Project. As evidenced by the many millions of systems that rely on the NTP server pool for time synchronisation, the NTP Pool Project successfully facilitates accurate time synchronisation at scale. However, as our results demonstrate, the pool's mechanisms for assigning time servers to NTP clients are vulnerable to hazardous attacks. We considered two attack strategies: (1) compromising existing NTP servers and (2) injecting new time servers into the NTP server pool, and show that both are alarmingly effective. 
An attacker in control over fairly few existing NTP servers in a country/ continent can impact time at NTP client across the entire country/continent. A NTP client that uses the NTP server po is periodically assigned time servers to sync to by the pool. We showed (Perry al., 2021) that even an attacker in control of fairly few servers in the pool can inflict significant harm. The root cause for this vulnerability is that the pool's mechanis for assigning time servers to clients oblivious to inter-server dependencies. Local time at most NTP servers is derived from interaction with other time servers. This implies that an attacker in control of a few NTP servers with great influence over other time servers can potentially manipulating time at other time servers (with whom the client communicates We showed, through extensive empiric We showed, the che extensive empiric analyses, how this can be leveraged by state-scale, or even continent-scale adversely impacting the performance or security of various applications. In particular, an attacker in control of merely 10 s of time servers in Europe or the US lout of thousands of time servers in Europe and many hundreds in the US) can shift time forward/backwards by hours at many clients across the entire continent/country, impacting various applications of interest. We observed however, that more effective and als simpler to launch attacks are feasible by injecting new time servers into the NTP server pool.

Influencing time computation at clients via the injection of new time server into the NTP server pool is effective and simple. Entering a new time server into the NTP server pool is remarkably easy. Moreover, she/he entering the new time server, and operating that time server, is about the time server. We showed how through the proper configuration of parameters, an attacker that enters a time server into the pool can increase the number of clients its time server is assigned to by the pool by three orders of magnitude (compared to the default time server configuration). As our empirical analyses established (Perry et al., 2021), this translates to hundreds of thousands of clients per hour trying to sync with that time server. We showed how this state of affairs can be leveraged by an attacker for launching large-scale opportunistic attacks (as with taking over existing NTP servers) and for launching strategic and stealthy attacks that target specific NTP clients. In addition, this attack strategy is not limited in terms of the extent to which time can be shifted and can be employed to shift time at clients by days weeks, months, and beyond, impacting

\section{Why is NTP so insecure?}

There are two crucial aspects of NTP that make it particularly susceptible to tim shifting attacks.

1. The mechanisms used by the NTP server pool to determine which time servers a particular NTP client should sync with. As discussed previously, the NTP pool's mechanisms for determining which time servers in the pool to assign to a client ignore potential dependencies between the assigned time servers, which enables an attacker in direct control of one time server assigned to the client to influence the local times at other time servers assigned to the client. In addition, the ease at which an attacker can inject disproportionately influential time servers into the pool enables an attacker to influence time at many NTP clients that query the pool for time servers to sync with.
2. The client-side scheme for inferring the current time from time serverprovided responses is highly susceptible to manipulation. As previously explained, NTP was not designed with strategic attackers in mind, but to provide accurate time in the presence of time servers with false times, assumed to be fairly rare and non-strategic. Consequently, the algorithm executed at the NTP client to detect which time servers (from those assigned to the NTP client by the server pool) provide false time reports, and to infer the correct time from the information reported by the remaining time servers, is highly vulnerable to powerful, strategic attackers.

\section{Putting an end to internet}

\section{time shifters}

We presented (Deutsch et al., 2018; Perry et al. 2021) a holistic approach for ecuring NTP from timeshifting attecks, wich comprises two elements

1. Ananke: a root-of-trust for internet time synchronisation. Ananke is a set of 100s of NTP servers that are carefully chosen, and periodically audited, to eliminate the possibility of inter-server dependencies and to significantly raise the bar for an attacker whose purpose is to inject malkious time servers into Ananke. (See: Perry et al., 2021.)

Chronos: a secure NTP client. A Chronos NTP client periodically queries small subsets (e.g. of size 10-15) of NTP servers from a large set of time servers, consisting of 100 s of time servers, to solicit timing information, and then applies a theory-informed algorithm to remove outliers and average over the remaining responses. We proved
(Deutsch et al., 2018) that, so long as the attacker cannot influence the loc times at a large fraction of the tim servers in a large time server set, th crowdsourcing scheme guarantees that the client's internal clock remain close (time-wise) to the universal tim (UTC) and that the clocks of any two Chronos-clients remain close to eac other. For instance, to shift time at Chronos client by over $100 \mathrm{~ms}$ from the Coordinated Universal Time, even a powerful attacker requires over 20 years of effort in expectation.

Putting Ananke and

Chronos together

Our objective is to enhance NTP's security against malicious time servers while not adversely impacting its time accuracy and precision, nor the distribution of load

位s time servers. Our solution is simple: each NTP client should simultaneousy run two parallel synchronisation processes. The first synchronisation process is precisely that used by today's NTP clients to sync with pool-assigned servers in their region. This 'primary' synchronisation process is used, by default, to determine the client's local time. The second synchronisation process is run in 'watchdog mode'; the client applies Chronos' provably secure time-inference scheme to the servers in Ananke. So long as the watchdog's (Chronos') time does not deviate from the primary time by 'too much', the primary synchronisation process continues to update the local time. If, however, the results of these two time calculations are too far apart, which is indicative of an attack, the watchdog takes over and updates the local time (until such a time when the two computed times are close again).

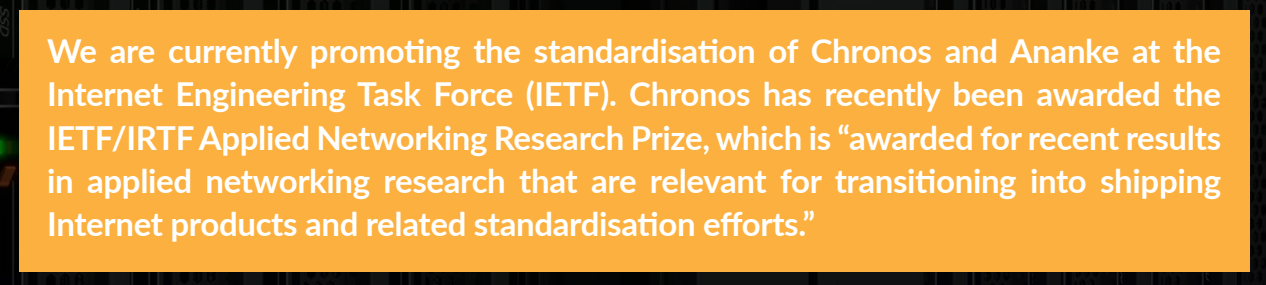

References

Deutsch, O., Schiff, N.R., Dolev, D. and Schapira, M. (2018) 'Preventing (Network) Time Travel ./Www.ndss-symposium.org/wp-content/uploads/2018/02/ ndss2018_02A-2_Deutsch_paper.pdf

Perry, Y., Schiff, N.R. and Schapira, M. (2021) 'A Devil of a Time: How Vulnerable is NTP to
Malicious Timeservers?', The Network and Distributed System Security (NDSS) Symposium 2021 Online, 21-25 February Available at: htttps://www.ndss-symposium.org/wp-content/uploa ndss2021_1A-2_24302_paper.pdf

\section{"We present a holistic approach for securing} NTP from time-shifting attacks."

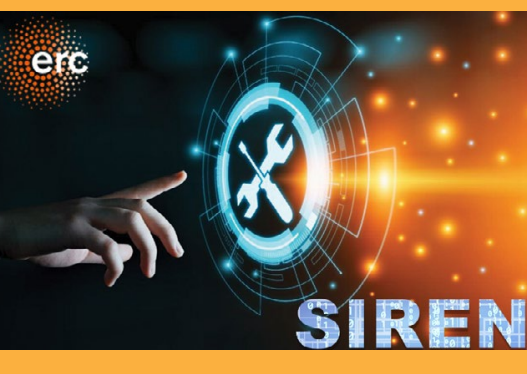

\section{PROJECT SUMMARY}
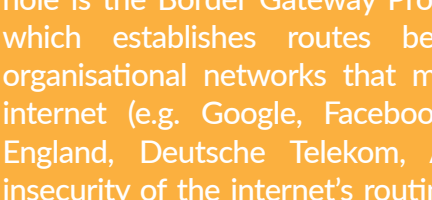$$
\text { . }
$$$$
\text { . }
$$

PROJECT LEAD



\section{.}

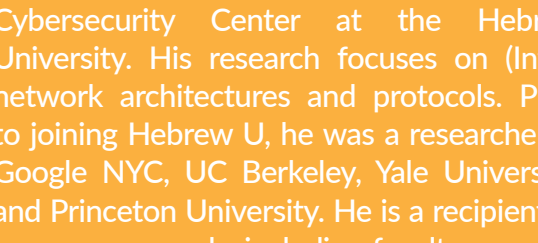

\section{.}

CONTACT DETALIS

Michael Schapira

III

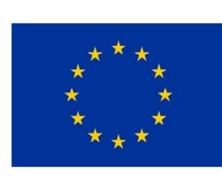

